\title{
IL-17 response mediates acute lung injury induced by the 2009 Pandemic Influenza A (H1N1) Virus
}

Chenggang $\mathrm{Li}^{1,{ }^{*} \text {, Penghui Yang }}{ }^{2,}$, Yang Sun ${ }^{1, *}$, Taisheng $\mathrm{Li}^{3,}{ }^{3}$, Chen Wang ${ }^{4,}$, Zhong Wang ${ }^{5, *}$, Zhen Zou ${ }^{1}$, Yiwu Yan ${ }^{1}$, Wei Wang ${ }^{1}$, Chen Wang ${ }^{1}$, Zhongwei Chen ${ }^{2}$, Li Xing ${ }^{2}$, Chong Tang ${ }^{2}$, Xiangwu Ju ${ }^{1}$, Feng Guo ${ }^{1}$, Jiejie Deng ${ }^{1}$, Yan Zhao ${ }^{1}$, Peng Yang ${ }^{1}$, Jun Tang ${ }^{1}$, Huanling Wang ${ }^{3}$, Zhongpeng Zhao ${ }^{2}$, Zhinan Yin ${ }^{6}$, Bin Cao ${ }^{4}$, Xiliang Wang ${ }^{2}$, Chengyu Jiang ${ }^{1}$

${ }^{I}$ State Key Laboratory of Medical Molecular Biology, Institute of Basic Medical Sciences, Chinese Academy of Medical Sciences, Center of Translational Medicine, Peking Union Medical College, Tsinghua University, Beijing 100005, China; ${ }^{2}$ State Key Laboratory of Pathogens and Biosecurity, Beijing Institute of Microbiology and Epidemiology, Beijing 100071, China; ${ }^{3}$ Department of Infectious Diseases, Peking Union Medical College Hospital, Chinese Academy of Medical Sciences \& Peking Union Medical College, Beijing 100730, China; ${ }^{4}$ Beijing Chao-Yang Hospital, Beijing Institute of Respiratory Medicine, Capital Medical University, Beijing 100020, China; ${ }^{5}$ Emergency Department, Peking Union Medical College Hospital, Chinese Academy of Medical Sciences \& Peking Union Medical College, Beijing 100730, China; ${ }^{6}$ State Key Laboratory of Medicinal Chemical Biology, College of Life Sciences, Nankai University, Tianjin 300071, China

The 2009 flu pandemic involved the emergence of a new strain of a swine-origin H1N1 influenza virus (S-OIV H1N1) that infected almost every country in the world. Most infections resulted in respiratory illness and some severe cases resulted in acute lung injury. In this report, we are the first to describe a mouse model of S-OIV virus infection with acute lung injury and immune responses that reflect human clinical disease. The clinical efficacy of the antiviral oseltamivir (Tamiflu) administered in the early stages of S-OIV H1N1 infection was confirmed in the mouse model. Moreover, elevated levels of IL-17, Th-17 mediators and IL-17-responsive cytokines were found in serum samples of S-OIV-infected patients in Beijing. IL-17 deficiency or treatment with monoclonal antibodies against IL17-ameliorated acute lung injury induced by the S-OIV H1N1 virus in mice. These results suggest that IL-17 plays an important role in S-OIV-induced acute lung injury and that monoclonal antibodies against IL-17 could be useful as a potential therapeutic remedy for future S-OIV H1N1 pandemics.

Keywords: cytokine; acute lung injury; S-OIV H1N1

Cell Research (2012) 22:528-538. doi:10.1038/cr.2011.165; published online 25 October 2011

\section{Introduction}

During the spring of 2009, a novel swine-origin influenza A (H1N1) virus (S-OIV) that caused human infections in Mexico, spread first to the United States and Canada, and then spread globally [1-3]. By August 1, 2010, before WHO Director-General Dr Margaret Chan

\footnotetext{
*These six authors contributed equally to this work. Correspondence: Chengyu Jiang ${ }^{\mathrm{a}}$, Xiliang Wang ${ }^{\mathrm{b}}$

${ }^{a}$ Tel: +86-10-65296908; Fax: +86-010-65276551

E-mail: jiang@pumc.edu.cn

bE-mail: xiliangw@126.com

Received 6 April 2011; revised 5 July 2011; accepted 27 July 2011; published online 25 October 2011
}

announced that the H1N1 influenza event had moved into the post-pandemic period, almost every country had reported laboratory-confirmed cases, with over 18449 total deaths (http://www.who.int/csr/don/2010_08_06/en/ index. html). This was the first influenza pandemic since 1968, and it caused acute respiratory illness and acute lung injury, especially in its severe form. Acute respiratory distress syndrome was reported to be the predominant cause for the reported deaths $[4,5]$. Recent media reports from England and Finland have suggested that this influenza virus might return. The Health Protection Agency reported that of the recent 214 confirmed deaths in the UK, 195 were infected with the 2009 H1N1 strain. The official China Xinhua News announced that the Center for Disease Control in China monitored 17025 influenza 
patients from December 27, 2010 to January 2, 2011 in Beijing and found that $23.08 \%$ were infected with S-OIV H1N1 (http://news.xinhuanet.com/politics/2011-01/10/ c $12963482 . \mathrm{htm})$. Altogether, these data suggest that the 2009 H1N1 strain is still circulating in the world.

Previous clinical reports indicated that hypercytokinemia is involved in the pathology of severe 2009 pandemic influenza [6-8]. However, the underlying molecular mechanism is unclear. To elucidate the molecular pathogenesis, an appropriate animal model is needed. Models of the 2009 pandemic influenza have been reported in mice, ferrets, guinea pigs and non-human primates [912]. The selection of an appropriate animal model that accurately reflects the disease and the protective immune response against infection in human has been a major challenge in influenza research. Mice are commonly used as animal models for influenza research because of the convenience of genetic modulation, though they are not ideal for transmission studies [9]. However, currently available mouse models are incapable of emulating human influenza-based disease and the immune responses to the 2009 pandemic influenza $[9,13,14]$. Therefore, we screened several influenza virus strains isolated in China during the 2009 pandemic. We found that an isolate termed A/Beijing/501/2009 (H1N1) (BJ501) caused severe respiratory disease in mice and that it may be appropriate for use in setting up a mouse model for influenza research (Figure 1).

\section{Results}

Mice infected with the Beijing 501 (BJ501) strain of SOIV H1N1 developed severe acute lung injury

Compared with seasonal influenza, H1N1 and the pandemic 2009 A/California/07/2009 (CA07) isolate, which does not cause mortality in mice at the highest possible virus titers, the BJ501 isolate had a median lethal dose (LD50) at a virus titer of $\log 104.2$ in 6- to 8-week-old $\mathrm{B} / \mathrm{C}$ mice (Figure 1A). Moreover, 4-week-old B6 mice were found to be more sensitive to the 2009 pandemic influenza infection and to show representative characteristics of the human disease (Figure 1A). This result is consistent with the clinical reports that infants and children, infected with influenza are more likely to develop complications than adults [5]. Therefore, 4-week-old B6 mice were used for the influenza model in this report. However, compared with the commonly used mouseadapted influenza A/Puerto Rico/8/34 (PR8) strain, the mortality rate caused by BJ501 was lower (Figure 1A). Mouse body weight loss induced by BJ501 infection was as high as $40 \%$, whereas the weight losses caused by seasonal $\mathrm{H} 1 \mathrm{~N} 1$ and CA07 isolate infections were $10 \%$ and 20\%, respectively (Figure 1B). However, no differences in virus replication were observed among strains (Figure 1C). The pathological alterations of mouse lung tissue infected with the 2009 pandemic influenza BJ501 strain were more severe than those in tissue infected with the 2009 pandemic influenza CA07 or seasonal influenza (Figure 1D). Lung edema, as defined by the wet-to-dry ratio of the lung tissue, was observed in BJ501 and PR8 infections (Figure 1E). These data of acute lung injury are consistent with the clinical reports of 2009 pandemic influenza [15]. Thus, 4-week-old B6 mice infected with the BJ501 isolate, whose hemagglutinin (HA) genome is 99\% identical to the 2009 pandemic CA07 strain (Figure $1 \mathrm{~F}$ ), could potentially be an ideal animal model for severe cases of 2009 pandemic influenza infection in hu$\operatorname{man}[16,17]$.

\section{Administration of Oseltamivir at early stages of infection ameliorated acute lung injury}

During the 2009 pandemic, no specific therapeutics existed for the treatment of the disease. The commonly applied therapeutics included antiviral (mostly Tamiflu/ Oseltamivir) and corticosteroid treatments [18]. While controversy regarding the drug efficacy of corticosteroids in the treatment of acute respiratory distress syndrome patients infected with 2009 pandemic H1N1 has been reported $[19,20]$, the effects of Tamiflu treatment at an early stage of disease-onset are well-established [21-23]. Using our newly established mouse model, infected with the 2009 pandemic BJ501 strain, we tested the drug efficacy of Oseltamivir. The survival rate of the mice was significantly improved when they were given Oseltamivir during the first 3 days of infection (Figure 2A). However, improvement in the survival rate was not significant when the mice were given Oseltamivir after the first 3 days of infection (Figure 2B). Acute lung injury, as measured by lung pathology and wet-to-dry weight ratio, was also improved when mice were given Oseltamivir in the early stages of BJ501 infection (Figure 2C, 2D). These results were consistent with the reported human clinical data [23]. Therefore, 4-week-old B6 mice, infected with the BJ501 isolate, represent a validated animal model that can be used to evaluate potential therapeutic drugs.

Cytokine responses in patients and mice infected with 2009 H1N1 influenza A virus

Previous clinical reports analyzing sera obtained from 29 patients in Spain and 57 patients in Hong Kong showed that Th-1 and Th-17 hypercytokinemia is an early host response signature in severe cases of 2009 pandemic influenza $[6,8]$. However, controversy regarding the role of the Th-17 response was raised by a 
A

\begin{tabular}{ccccc}
\hline & \multicolumn{4}{c}{ LD $_{50}$} \\
\cline { 2 - 5 } & $\begin{array}{c}\text { Seasonal } \\
\text { (H1N1) }\end{array}$ & $\begin{array}{c}\text { A/Beijing/501/2009 } \\
\text { (H1N1) }\end{array}$ & $\begin{array}{c}\text { A/California/07/20099 } \\
\text { (H1N1) }\end{array}$ & $\begin{array}{c}\text { A/PuertoRico/8/1934 } \\
\text { (H1N1) }\end{array}$ \\
\hline C57 BL/6: 4 weeks & $>10^{5.5}$ & $10^{1.9}$ & $10^{3.5}$ & $<10^{1.5}$ \\
\hline C57 BL/6: 6-8 weeks & $>10^{5.5}$ & $10^{3}$ & $>10^{5.5}$ & $<10^{1.5}$ \\
\hline BALB/c: 4 weeks & $>10^{5.5}$ & $10^{3.8}$ & $>10^{5.5}$ & $10^{1.6}$ \\
\hline BALB/c: 6-8 weeks & $>10^{5.5}$ & $10^{4.2}$ & $>10^{5.5}$ & $10^{2.8}$ \\
\hline
\end{tabular}

B



D

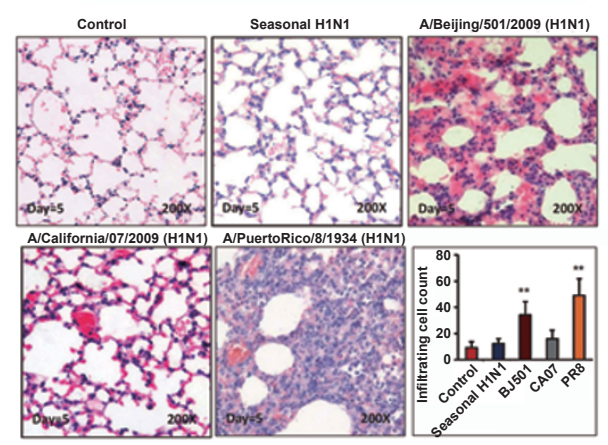

C

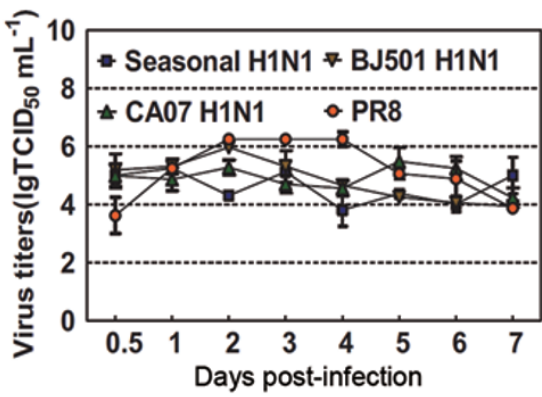

E

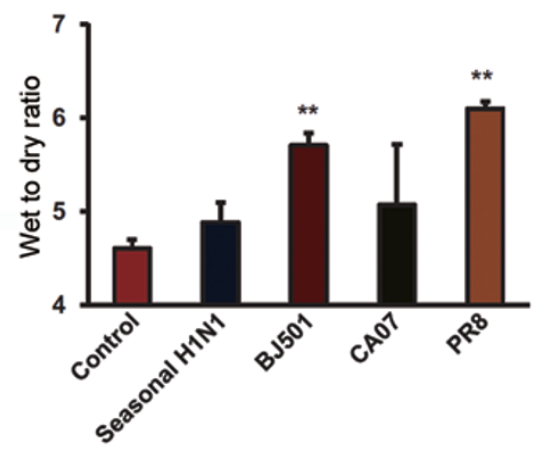

F

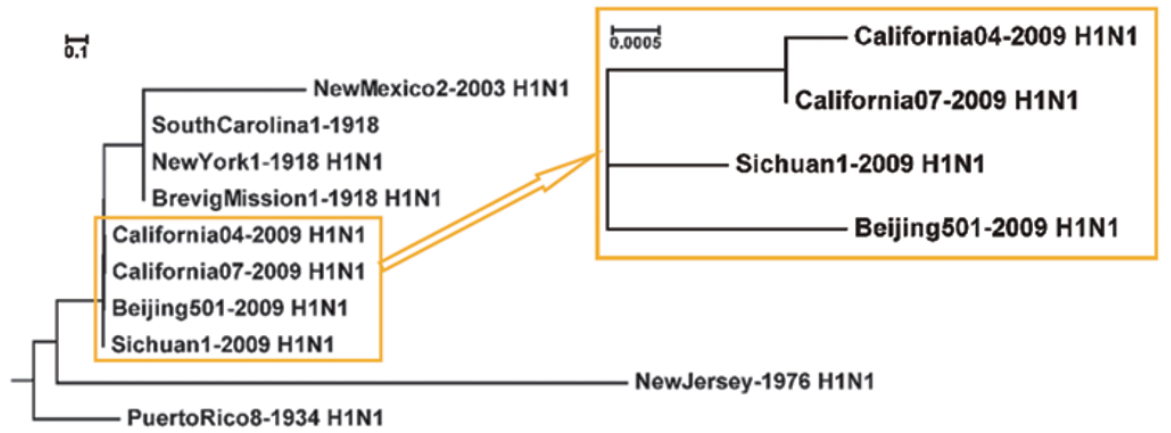

Figure 1 The BJ501 strain of S-OIV H1N1-induced acute lung injury in mice. (A) LD50 of influenza A (H1N1) viruses in 4and 6-to-8-week-old B/C and B6 mice. For (B-E), 4-week-old B6 mice were infected with 105.5 TCID50 of Control, Seasonal, BJ501, CA07, or PR8. (B) Changes in body weight. The values are Means \pm SEM from five mice. (C) Growth properties of viruses. At the indicated times, lung tissues were collected, and virus titers were determined by plaque assays in MDCK cells. The values are Means \pm SEM from three mice. (D) Histological findings from lung tissues. Representative pathological images for the lungs of mice at $5 \mathrm{DPI}$. The mean numbers of infiltrating cells per microscopic field \pm SEM are shown $(n=100$ fields analyzed for three mice per group). Magnification $=200 \times$. (E) Wet-to-dry ratios of lungs at 5 DPI. (F) A phylogenetic tree of HA genes of the influenza A $(\mathrm{H} 1 \mathrm{~N} 1)$ virus was constructed using the Maximum Likelihood and Bayesian-tree methods by comparing seasonal influenza A virus strains A/PuertoRico8/1934 (PR8), A/New Jersey/1976, A/New Mexico/2/2003 and A/South Carolina/1/1918, A/New York/1/1918, and A/Brevig Mission/1/1918 with the pandemic influenza A virus strains A/ California/04/2009, A/California/07/2009, A/Beijing/501/2009 and A/Sichuan/1/2009 from early 2009. The values are means \pm SEM from six mice. ${ }^{*} P<0.05$ and ${ }^{* *} P<0.01$. 
A
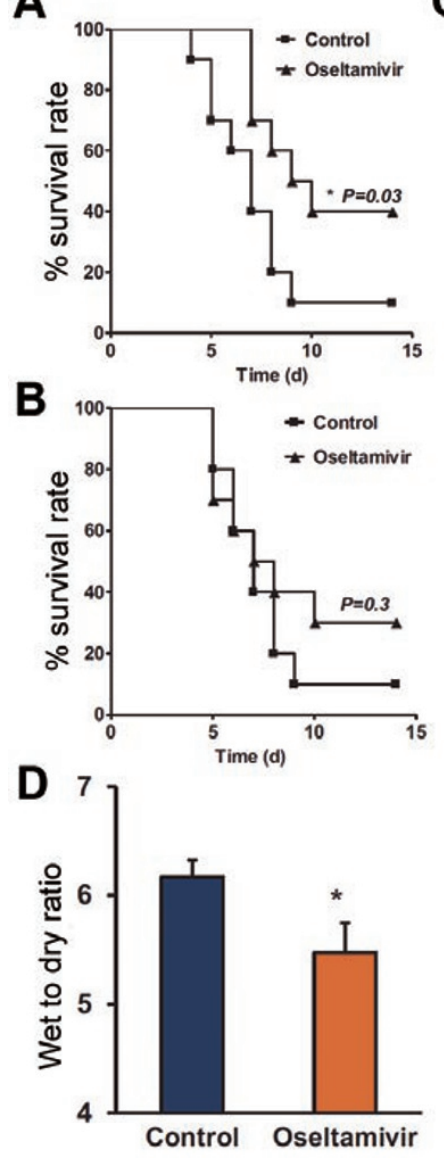

Figure 2 Administration of Oseltamivir phosphate during early infection ameliorated acute lung injury in mice. B6 mice were treated intraperitoneally with control or $10 \mathrm{mg}$ per $\mathrm{kg}$ per day with Oseltamivir phosphate. (A) Mortality rates of BJ501-infected B6 mice $(n=10)$ treated for 3 successive days. (B) Mortality rates of $\mathrm{B} 6$ mice treated for 10 successive days, 48 hours after infection with BJ501 ( $n=10)$. (C) Histological findings from lung tissues. Representative pathological images at $5 \mathrm{DPI}$ from the lungs of mice infected with 105.5 TCID50 of BJ501 and treated for 3 successive days. The mean numbers of infiltrating cells per microscopic field \pm SEM are shown ( $n=100$ fields analyzed for three mice per group). Magnification $=200 \times$. (D) Wet-to-dry ratios of lungs at $5 \mathrm{DPI}$ from mice infected with 105.5 TCID50 of BJ501 and treated for 3 successive days. The values are means \pm SEM from six mice. ${ }^{*} P<0.05$ and ${ }^{* *} P<0.01$.

recent study from a Romanian group reporting cytokine responses in 32 patients with severe H1N1 influenza A virus infection [7]. The elevation of IL-17 was observed in neither mild nor acute respiratory distress syndrome cases [7]. In an effort to further clarify this issue, we recruited 37 patients who were PCR positive for the 2009 H1N1 influenza A virus from two hospitals in Beijing. The characteristics underlying the conditions and out-

comes of the 37 patients infected with 2009 influenza A are described in Supplementary information, Table S1. After excluding patients whose serum samples were collected 8 days after disease-onset, patients under 16 years old and patients who had immune diseases (Supplementary information, Figure S1), we profiled 24 cytokines and chemokines from the serum samples of 23 qualified patients (Figure 3A). Unlike previous reports, Th-1 hypercytokinemia was not observed in our patient groups compared with healthy individuals [6] (Figure 3A). However, IL-17 was elevated in all mild, hospitalized and critical patient groups (Figure 3A). Th-17 mediators, including IL-6 and IL-8, and IL-17-responsive cytokines and chemokines, including G-CSF and GM-CSF, were also elevated (Figure 3A), suggesting that IL-17 may play an important role in the pathogenesis associated with 2009 pandemic influenza infection. Using the B6 mouse model infected with the BJ501 virus, we profiled 23 mouse cytokines and chemokines from the mouse lung bronchoalveolar lavage fluids (BALFs) (Figure 3B). The profile of cytokines and chemokines from infected mice was generally similar to that from patient serum samples in Beijing, indicating that 4-week-old B6 mice infected with the BJ501 isolate could reflect human immune responses to 2009 pandemic influenza infection in the Beijing area. Levels of IL-17, Th-17 mediators, and IL-17-responsive cytokines and chemokines were all elevated in the BALFs of infected mouse lungs (Figure 3B). We thus hypothesized that IL-17 might play a critical role in the host response to 2009 pandemic influenza infection.

IL-17-deficient mice ameliorated acute lung injury induced by the BJ501 strain of S-OIV H1N1

The Th-17 hypothesis is the first major revision of the Th-1/Th-2 hypothesis of T cell-mediated tissue damage [24]. IL-17 has been associated with tissue damage in the brain, joints, heart, lungs and intestines, in experimental models [25-27]. However, the role of IL-17 in lung injury induced by influenza is controversial [28-30]. Here, the establishment of a suitable mouse model enabled us to directly test whether IL-17 mediates or protects from the acute lung injury induced by 2009 pandemic influenza infection.

To this end, IL-17A-deficient mice were infected with the 2009 pandemic influenza virus BJ501. We found that the survival rates of mice were significantly increased compared with infected wild-type (WT) mice (Figure $4 \mathrm{~A})$. Body weight losses of $>30 \%$ were observed in infected WT control mice, but were not seen in infected IL-17A-deficient mice (Figure 4B). Compared with the infected WT control mice, the lung histopathologies 

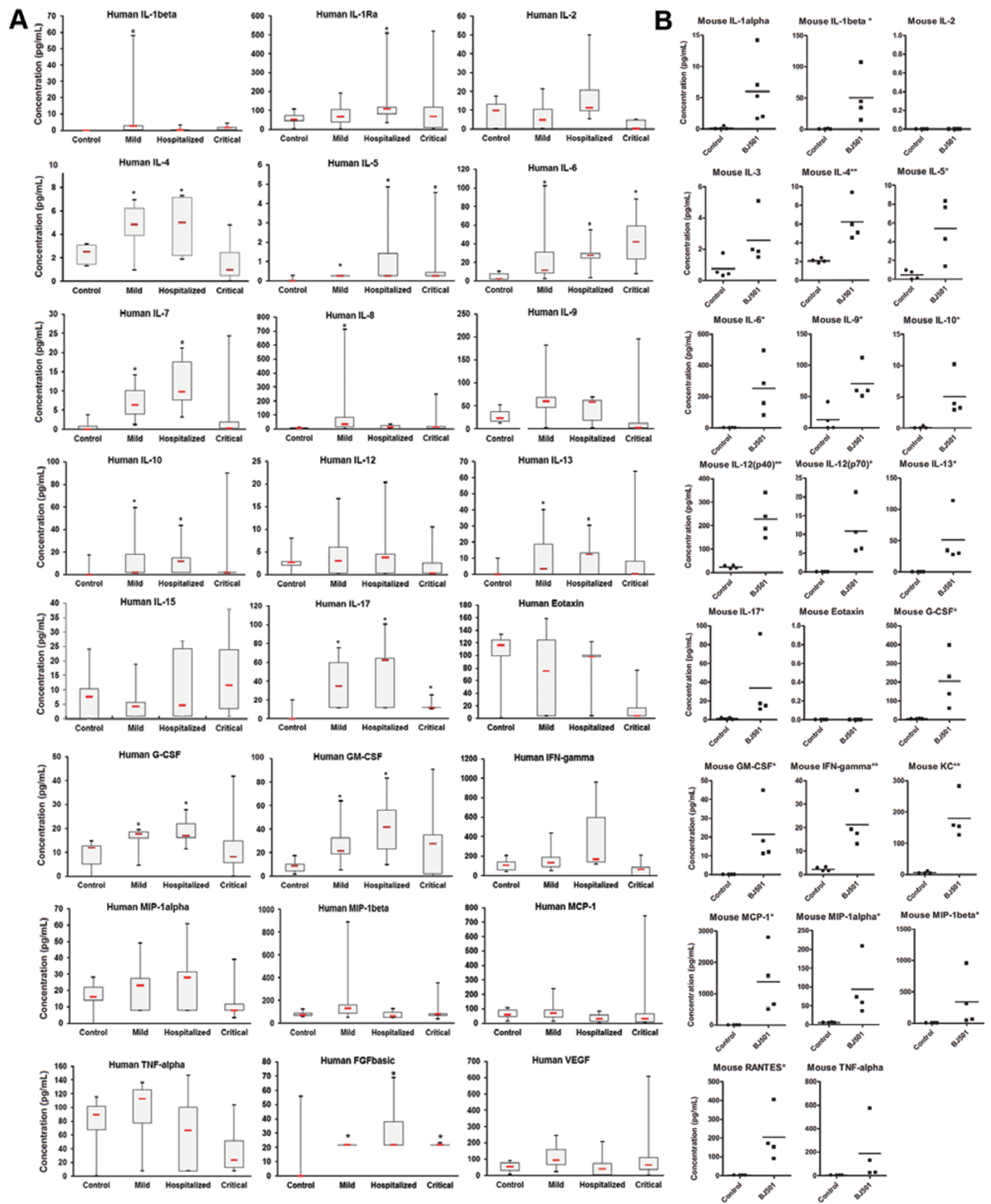

Figure 3 Profiles of cytokines and chemokines in serum samples of Beijing patients infected with S-OIV H1N1 and in BALF samples of BJ501-infected mice. (A) Cytokine concentrations in sera from patients confirmed to have influenza A H1N1 virus infection were determined using a Bio-Plex Human Cytokine 25-Plex Array. (B) Cytokine concentrations in BALFs taken at 24 hours post-infection from B6 mice infected with 105.5 TCID50 of BJ501 or the control were determined using a Bio-Plex Mouse Cytokine 23-Plex Array. ${ }^{*} P<0.05$ and ${ }^{* *} P<0.01$. 
A



B

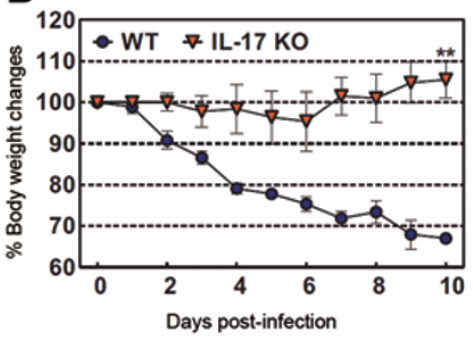

C


$\mathbf{E}$

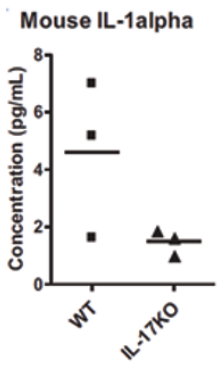

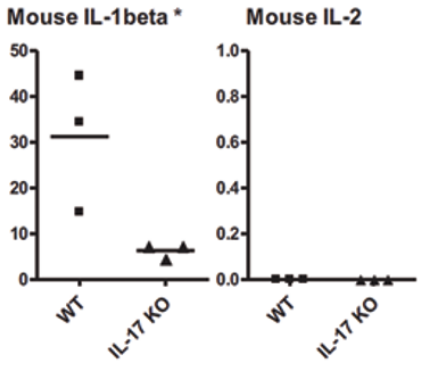
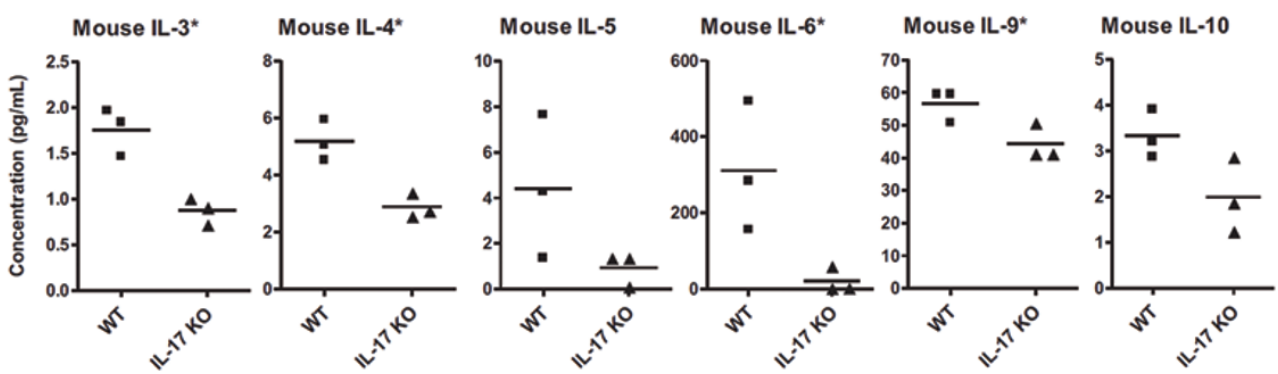

Mouse IL-12(p40)**
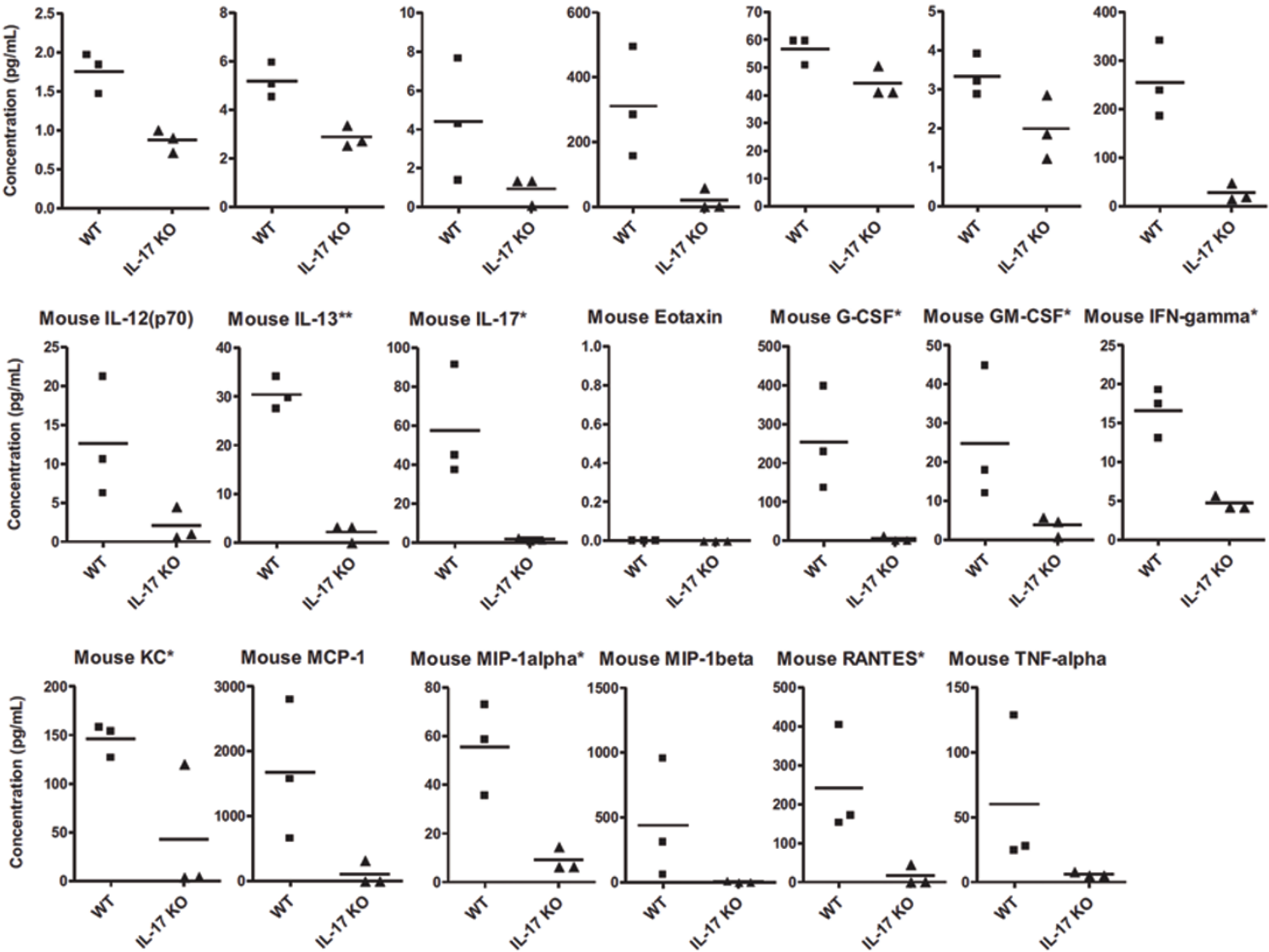

Figure $4 \mathrm{IL}-17^{-1-}$ mice ameliorated acute lung injury induced by S-OIV. WT B6 mice and IL-1 $17^{-1-}$ mice (B6 background) were intranasally inoculated with 105.5 TCID50 of BJ501. (A) Survival rates of WT B6 mice and IL-17 $7^{-1-}$ mice $(n=10)$. (B) Changes in body weights. The values are means \pm SEM from ten mice. (C) Histological findings from lung tissues. Representative pathological images from the lungs of WT and IL-17 $7^{-1-}$ mice at $5 \mathrm{DPI}$. The mean numbers of infiltrating cells per microscopic field \pm SEM are shown ( $n=100$ fields analyzed for three mice per group). Magnification $=200 \times$. (D) Wet-to-dry ratios of lungs at $5 \mathrm{DPI}$. The values are means \pm SEM from six mice. (E) Concentrations of specific inflammatory cytokines in the BALFs of WT B6 and IL-1 $17^{-1-}$ mice (B6 background) at 24 hours post-infection were determined using a Bio-Plex Mouse Cytokine 23Plex Array. ${ }^{*} P<0.05$ and ${ }^{* *} P<0.01$. 
in IL-17A-deficient mice were significantly improved, and lung injury scores, as defined by leukocyte infiltration cell counts, were significantly reduced (Figure 4C). Moreover, the wet-to-dry lung weight ratios used to measure pulmonary edema in IL-17A-deficient mice were significantly decreased (Figure 4D). Notably, the levels of Th-17 mediators and responsive cytokines and chemokines were all significantly reduced in the infected IL-17A-deficient mice compared with infected WT control mice (Figure 4E). Altogether, these data indicate that IL-17 plays a critical role in mediating the acute lung injury caused by 2009 pandemic H1N1 influenza infection.

Monoclonal antibodies against IL-17 protected against acute lung injury in S-OIV H1N1-infected mice

Our finding that the IL-17 response to 2009 pandemic H1N1 influenza plays a critical role in mediating acute lung injury not only sheds light on the molecular mechanism of acute lung injury induced by 2009 pandemic H1N1 influenza, but also suggests that inhibition of IL17A may therapeutically ameliorate the induced acute lung injury. Currently, therapeutic monoclonal antibodies comprise the majority of recombinant proteins used in clinics [31]. To test whether monoclonal antibodies against IL-17A could protect against 2009 pandemic influenza infection, we treated BJ501 virus-infected mice with monoclonal antibodies against human IL-17A. We found that the therapeutic effect of the monoclonal antibody against IL-17A was significant. The survival rates of mice treated with anti-IL-17A monoclonal antibodies were significantly increased compared with isotype control-treated mice (Figure 5A), and body weight losses in mice treated with anti-IL-17A monoclonal antibodies were reduced (Figure 5B). In addition, lung histopathology alterations and formation of lung edema in the infected mice treated with anti-IL-17A monoclonal antibodies were significantly improved compared with the isotype control-treated mice (Figure 5C and 5D). The levels of Th-17 mediators and responsive cytokines and chemokines were also all significantly reduced in the infected mice treated with anti-17 antibodies compared with infected mice treated with isotype-control (Figure $5 \mathrm{E})$.

IL-17 could be secreted by many cells, such as Th17 cells, gamma delta $T$ cells and natural killer cells [32, 33]. Cytokine kinetics of mice BALFs infected with BJ501 virus were assessed. The elevation of both IL-17A and IL-17F were detectable at $12 \mathrm{~h}$ of post-infection, suggesting that gamma delta $\mathrm{T}$ cells may be the main source of IL-17 elevation (Figure 6A, 6B).

We also tested whether inhibition of Th- 1 mediators provides protection against 2009 pandemic virus infec- tion. Clinically available monoclonal antibodies against TNF-alpha receptor II were injected to mice infected with BJ501, and the survival rates of these monoclonal antibody-treated mice were not improved (Supplementary information, Figure S2A). Body weight losses of 30\% were observed in these mice (Supplementary information, Figure S2B), and lung tissue wet-to-dry ratios also were not improved, compared with isotype-control-treated mice (Supplementary information, Figure S2C). This finding is consistent with a previous report that TNFR signaling was dispensable for weight loss in response to PR8 virus infection [29]. In addition, we monitored virus replication in infected lungs of mice treated with monoclonal and isotype control antibodies. No difference in virus replication was observed, indicating that IL-17 may not influence the proliferation of BJ501 influenza (Supplementary information, Figure S3). Altogether, these data suggest that therapeutic anti-IL-17A monoclonal antibody treatment may be a useful remedy in the event of a return of the 2009 influenza pandemic.

\section{Discussion}

The role of IL-17 was previously investigated in animal models of PR8 influenza infection. The role of IL17 as a protective cytokine against lethal influenza challenge and its role in mediating the immunopathology of influenza infection have been reported [28, 29]. Hamada et al. [28] claimed that IL-17-secreting CD4 T (Th)-17 and CD8 T (Tc)-17 effector cells were present in the lung following primary challenge with influenza $\mathrm{A}$, and reported that a blocking-antibody treatment against IL-17 increased weight loss and reduced survival rate. In reality, the anti-IL-17 antibody studies were only performed in a heterotypic challenge model. The authors did not study anti-IL-17 in primary influenza infection, in which nearly all of the IL-17 comes from gamma delta T cells [28]. Crowe et al. [29] treated IL-17RA-deficient mice with influenza $\mathrm{A}$, and found that weight loss and survival rates were improved in IL-17RA-deficient mice. Three ligands, IL-17A, IL-17F, and IL-25, have been reported capable of signaling through IL-17RA [34]. Previous report demonstrated that IL-17F and IL-25 played opposing roles of IL-17A in the regulation of lymphopoiethin production in human nasal epithelial cells [35]. Our study found that IL-17F was also elevated in BALF of mice infected with BJ501 live virus (Figure 6B). Further studies are necessary to elucidate the roles of IL-17F and IL-25 in the BI501 virus infection. The role of IL-17 is more complicated when bacterial pneumonia is studied in the context of influenza A co-infection. Kudva et al. [30] found that influenza A inhibits the Th-17-mediated 
A

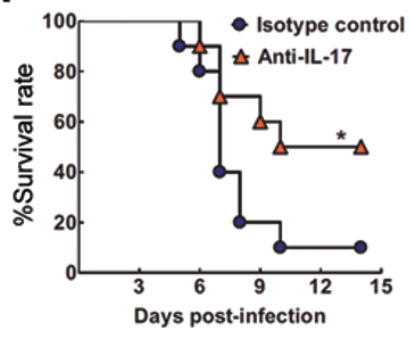

D

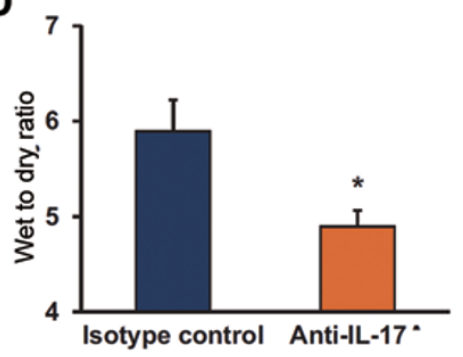

B



C
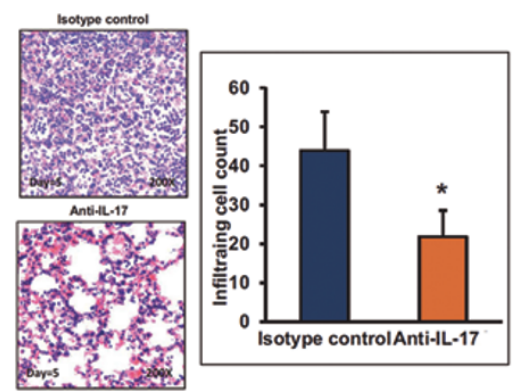

$\mathbf{E}$
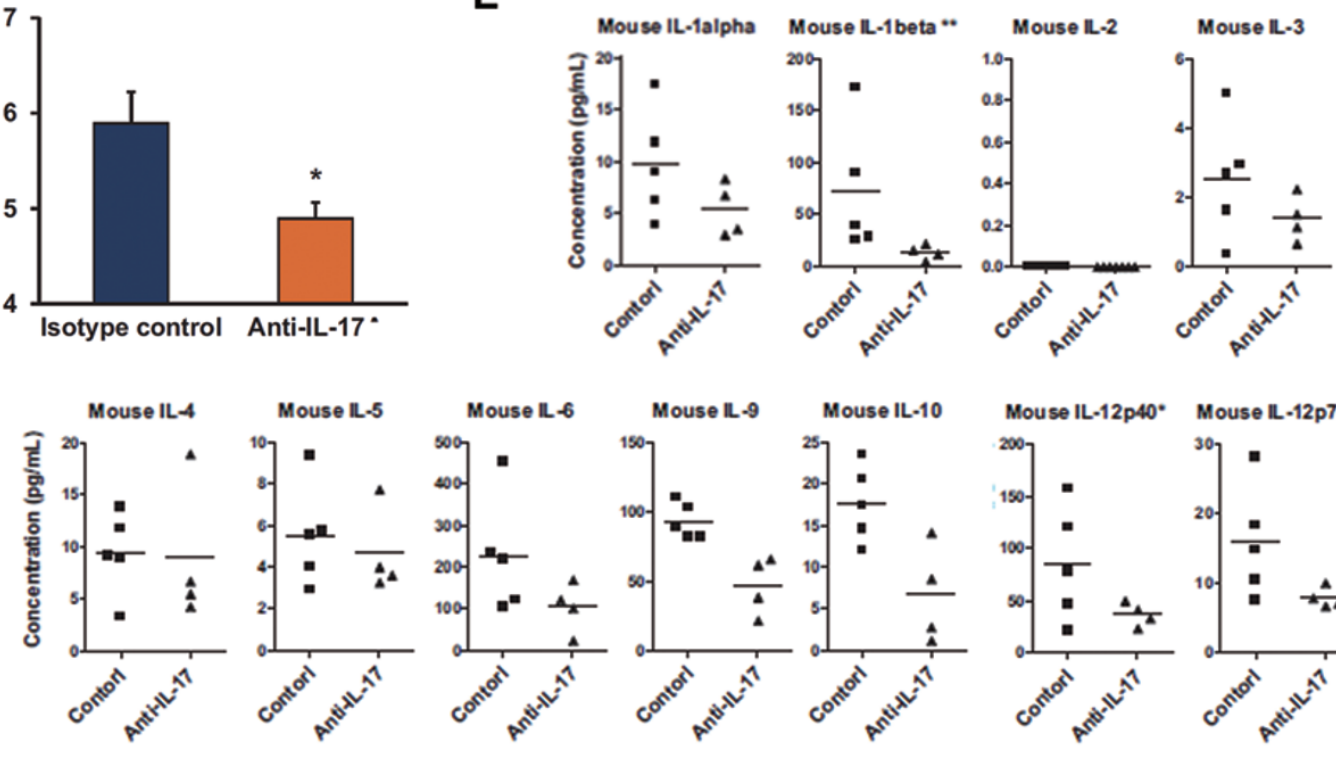

Mouse IL-10
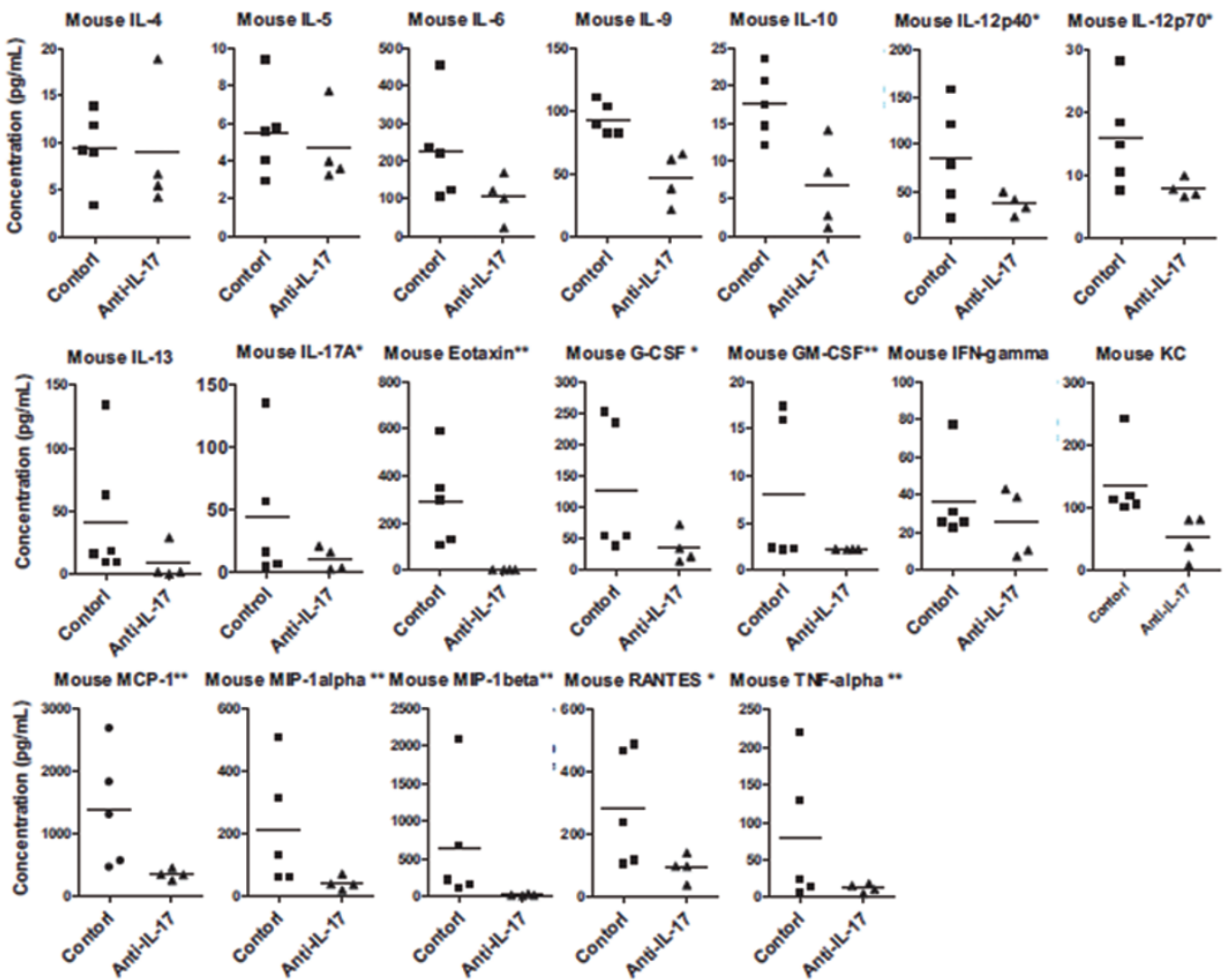

Figure 5 Monoclonal antibodies against IL-17 ameliorated S-OIV H1N1-induced acute lung injury in mice. B6 mice were treated intravenously with $50 \mathrm{\mu g}$ of isotype-control or anti-IL-17 antibodies per mouse 24 hours before infection and at 1 day and 3 DPI. (A) Mortality rates of BJ501-infected B6 mice $(n=10)$. (B) Changes in the body weights of B6 mice $(n=10)$. (C) Histological findings from lung tissues. Representative pathological images for the lungs at 5 DPI of mice infected with 105.5 TCID50 of BJ501 and intravenously treated with isotype-control or anti-IL-17 antibodies. The mean numbers of infiltrating cells per microscopic field \pm SEM are shown $(n=100$ fields analyzed for three mice per group). Magnification $=200 \times$. (D) Wet-to-dry ratios of lungs at 5 DPI with BJ501 at 105.5 TCID50. The values are means \pm SEM from six mice. ${ }^{*} P<0.05$ and ${ }^{* *} P<0.01$. (E) Concentrations of specific inflammatory cytokines in the BALFs of BJ501-infected B6 mice at 24 hours postinfection were determined using a Bio-Plex Mouse Cytokine 23-Plex Array. ${ }^{*} P<0.05$ and ${ }^{* *} P<0.01$. 

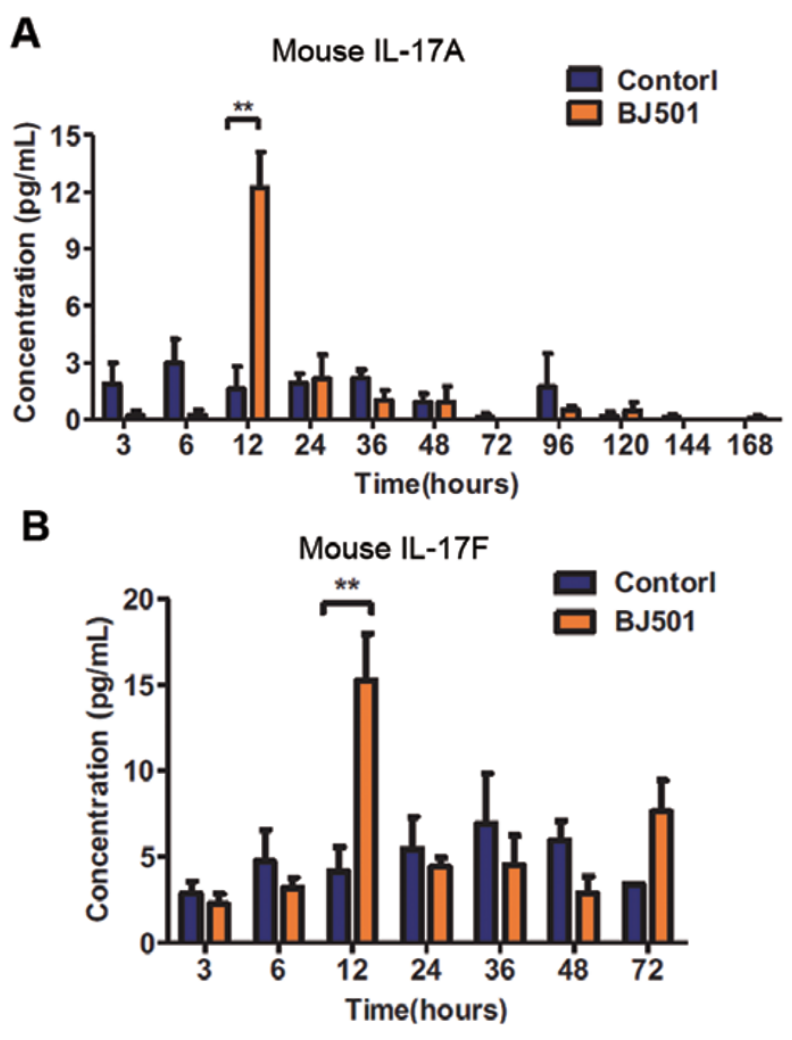

Figure 6 Cytokine kinetics of IL-17A and IL-17F in BALF samples from BJ501-infected mice. (A) IL-17A and (B) IL-17F concentrations in the BALFs of B6 mice infected with BJ501 at 105.5 TCID50 or the control were determined using a Bio-Plex suspension system. ${ }^{* *} P<0.01$.

host defense against bacterial pneumonia in mice. Although further studies are necessary to elucidate the complex role of IL-17 in PR8 influenza infection, our SOIV infection studies support the hypothesis that IL-17 mediates acute lung injury in mice.

IL-17 has been shown to play critical roles in the immune response and be associated with various models of immune-mediated tissue injury [24, 33]. IL-17 acts as a pro-inflammatory cytokine that induces the expansion and accumulation of neutrophils of the innate immune system and links innate and adaptive immunity [33]. Our mouse model of the BJ501 strain of S-OIV H1N1 showed a cytokine profile similar to that observed in patient serum samples (Figure 3B). More studies using the mouse model of BJ501 S-OIV H1N1 infection will be necessary to further elucidate this mechanism.

A therapeutic, fully human monoclonal antibody against IL-17 (mAb AIN457) has been developed by Novartis, and Phase II and Phase III clinical trials for uveitis, Crohn's disease, psoriasis and rheumatoid arthritis are underway $[36,37]$. The clinical data for the mono- clonal antibody AIN457 and placebo groups support the role of IL-17 in the pathophysiology of inflammatory diseases, including uveitis, psoriasis and rheumatoid arthritis [37]. Our experimental results from the mouse model of S-OIV H1N1 infection suggest that such therapeutic approaches could be useful for patients infected with the 2009 pandemic influenza virus, which will likely be circulating in the winter of 2011/2012.

\section{Materials and Methods}

\section{Viruses and reagents}

The influenza viruses used in this study were seasonal H1N1, influenza A (H1N1) virus strain A/Beijing/501/2009 (BJ501), influenza A (H1N1) virus strain CA07, and influenza A (H1N1) virus strain A/PR/8/1934 (PR8). Live-virus experiments were performed in Biosafety Level 3 facilities under governmental and institutional guidelines. Viruses were propagated by inoculation into 10 - to 11-day-old SPF embryonated fowl eggs via the allantoic route.

Oseltamivir phosphate was purchased from Beijing HuameiHuli Biochem. Isotype-control antibody Mouse IgG2b was purchased from Beijing Protein Innovation, and anti-IL-17 antibody Mouse $\operatorname{IgG} 2 \mathrm{~b}$ was purchased from Beijing Protein Innovation.

Mice

WT 4- and 6- to 8-week-old BALB/c (B/C) and C57BL/6 (B6) mice (Vital River, Beijing) and 4-week-old IL-17 ${ }^{-/}$mice (B6 background, courtesy of Professor Yin) were used in this study in the animal facility at the Institute of Basic Medical Sciences of Peking Union Medical College in accordance with local and institutional guidelines. Detailed procedures are provided below.

\section{Viral pathogenesis in mice}

LD50 WT 4- and 6- to 8-week-old B/C and B6 mice were used in this study. Ten mice per group were anesthetized with pentobarbital sodium and intranasally inoculated with 101.5, 102.5, 103.5, $104.5,105.5$ or 106.5 of the $50 \%$ tissue culture infectious dosage (TCID50) of the virus or virus dilution buffer (diluent). Survival was monitored daily for 14 days, and the median lethal doses in the mice were calculated.

\section{Survival rate and body weight changes}

WT 4-week-old B6 mice were treated intranasally with 105.5 TCID50 of virus or virus diluent. The survival percentages and body weights in each group of ten mice were monitored daily for 14 days. Survival data were analyzed by Kaplan-Meier survival analysis using GraphPad Prism 5 software.

\section{Growth kinetics of the virus in the lung tissue of mice}

WT 4-week-old B6 mice were intranasally inoculated with 105.5 TCID50 of virus. Samples of lung tissue were collected at 6 , $24,48,72,96,120,144$ and 168 hours after infection. The titers of viruses in the lung tissue in each group of three mice were determined by plaque assay in MDCK cells.

\section{Histological examination}

After being anesthetized with pentobarbital sodium, 4-weekold B6 mice were treated intranasally with 105.5 TCID50 of virus 
or virus diluent and were then killed at various days post-infection (DPI). The lungs of each group of three mice were fixed in formalin, and then embedded in paraffin. Ultrathin sections were obtained and stained with hematoxylin-eosin. The inflammatory cells were counted and represented as cell numbers per $100 \times$ field.

\section{Acute pulmonary edema (wet-to-dry ratio)}

To assess the extent of acute pulmonary edema, the lung wetto-dry weight ratios were calculated. WT 4- and 6-to-8-week-old $\mathrm{B} / \mathrm{C}$ and B6 mice were used in this study. Six mice per group were anesthetized with pentobarbital sodium, and intranasally inoculated with 105.5 TCID50 virus or virus diluent. At 5 DPI, the wet weights of the lungs from the mice were measured. The lungs were then heated to $68^{\circ} \mathrm{C}$ for 24 hours, and the dry weights of the lungs were recorded, after which the wet-to-dry ratios were calculated.

\section{Cytokine and chemokine measurement}

Three WT 4-week-old B6 mice per group were used in this study. For cytokine and chemokine measurements, mouse BALFs were collected after the mice were inoculated with virus or virus diluent for 24 hours. BALFs were processed with the Bio-Plex Mouse Cytokine 23-Plex. Array analysis was performed using the Bio-Plex Protein Array system (Bio-Rad Laboratories).

\section{Antiviral sensitivity of viral infection in mice}

WT 4-week-old B6 mice were anesthetized with pentobarbital sodium and were infected intranasally with 105.5 TCID50 of BJ501. Oseltamivir phosphate or placebo was subsequently administered intraperitoneally: (1) oseltamivir phosphate: $10 \mathrm{mg}$ per $\mathrm{kg}$ per day, dissolved in $100 \mu \mathrm{l}$ of phosphate buffered saline and administered daily (starting at 6 hours before virus infection) for 3 days; (2) placebo: $100 \mu$ l of phosphate buffered saline, administered daily (starting at 6 hours before virus infection) for 3 days; (3) Oseltamivir phosphate: $10 \mathrm{mg}$ per $\mathrm{kg}$ per day, dissolved in 100 $\mu 1$ of phosphate buffered saline and administered daily (starting at 48 hours after virus infection) for 10 days; (4) placebo: $100 \mu \mathrm{l}$ of phosphate buffered saline, administered daily (starting at 48 hours after virus infection) for 10 days. Survival rate, histology and acute pulmonary edema (wet-to-dry ratio) were measured as described in the viral pathogenesis in mice section.

\section{Pathogenesis of the virus in $\mathrm{IL}-17^{-/}$mice}

WT 4-week-old B6 mice and IL-17 $7^{-1}$ mice (B6 background) were anesthetized with pentobarbital sodium and were intranasally inoculated with 105.5 TCID50 of BJ501 or an equal volume of virus diluent. The survival rate, body weight change, histology, acute pulmonary edema (wet-to-dry ratio), and cytokine and chemokine measurements were performed as described above in the viral pathogenesis in mice section.

\section{Anti-inflammatory sensitivity of viral infection in mice}

To test the sensitivity of the viral infection to treatment with anti-IL-17 antibodies and isotype control antibodies, WT 4-week-old B6 mice were anesthetized with pentobarbital sodium and were infected intranasally with 105.5 TCID50 of BJ501. Subsequently, anti-IL-17 antibodies or isotype control antibodies were intravenously administered ( $50 \mu \mathrm{g}$ per mouse each day for 3 administrations, starting 1 day before infection): (1) isotype control antibody or (2) anti-IL-17 antibody. Survival rate, body weight change, histology, acute pulmonary edema (wet-to-dry ratio) and cytokine and chemokine measurements were performed as described above in the viral pathogenesis in mice section.

\section{Cytokine and chemokine measurement in patient sera}

For cytokine and chemokine measurements, the sera of patients positive for influenza A (H1N1) virus infection were collected. Samples were processed with the Bio-Plex Human Cytokine 25Plex array. An array analysis was performed using the Bio-Plex Protein Array system (Bio-Rad Laboratories).

\section{Phylogenetic analyses of the HA gene segment}

The gene sequences encoding the surface protein HA of selected influenza A (H1N1) virus strains of A/Puerto Rico/8/1934 (PR8), A/New Jersey/1976, A/New Mexico/2/2003, A/South Carolina/1/1918, A/New York/1/1918, A/Brevig Mission/1/1918, A/California/04/2009, CA07, A/Beijing/501/2009, and A/Sichuan/1/2009 were collected from the website of the National Center for Biotechnology Information (NCBI) (http://www.ncbi.nlm.nih. gov/Taxonomy/Browser /wwwtax.cgi?mode=Tree\&id=114727\&1 $\mathrm{vl}=3 \&$ lin $=\mathrm{f} \& \mathrm{keep}=1 \&$ srchmode $=1 \&$ unlock). The sequences were aligned using the program MEGA v5.0, and the phylogenetic trees were reconstructed with MEGA v5.0 using the Maximum Likelihood and Bayesian-tree methods.

\section{Statistical analyses}

All data are shown as means \pm SEM. Measurements at single timepoints were analyzed by an ANOVA, and if they demonstrated significance, they were further analyzed by a two-tailed $t$-test. Time courses were analyzed by a repeated measurement (mixed model) ANOVA with Bonferroni post- $t$-tests. Survival data were analyzed by a Kaplan-Meier survival analysis. All statistical tests were conducted using GraphPad Prism 5 software. $P<0.05$ indicates statistical significance.

Other supplementary Materials and Methods refer to Supplementary information, Data S1.

\section{Acknowledgments}

We thank Zongsheng Han, Yunli Ling and Ran Zhang for technical support. This work was supported by the National Natural Science Foundation of China (30625013 and 30623009), the Ministry of Science and Technology of China (2009CB522102 and 2009CB522105) and the Ministry of Health (2009ZX10004-308). Dr Zhinan Yin was supported by the National Outstanding Young Scientist Award of National Natural Science Foundation of China (30725015).

\section{References}

1 Hamilton K. The emergence of the pandemic A/H1N1 2009 virus and its characteristics. Bull Mem Acad R Med Belg 2009; 164:260-263.

2 Smith GJ, Vijaykrishna D, Bahl J, et al. Origins and evolutionary genomics of the 2009 swine-origin H1N1 influenza A epidemic. Nature 2009; 459:1122-1125.

3 Chowell G, Bertozzi SM, Colchero MA, et al. Severe respiratory disease concurrent with the circulation of H1N1 influenza. N Engl J Med 2009; 361:674-679. 
4 Dominguez-Cherit G, Lapinsky SE, Macias AE, et al. Critically Ill patients with 2009 influenza $\mathrm{A}(\mathrm{H} 1 \mathrm{~N} 1)$ in Mexico. JAMA 2009; 302:1880-1887.

5 Louie JK, Acosta M, Winter K, et al. Factors associated with death or hospitalization due to pandemic 2009 influenza A(H1N1) infection in California. JAMA 2009; 302:18961902.

6 Bermejo-Martin JF, Ortiz de Lejarazu R, Pumarola T, et al. Th1 and Th17 hypercytokinemia as early host response signature in severe pandemic influenza. Crit Care 2009; 13:R201.

7 Hagau N, Slavcovici A, Gonganau DN, et al. Clinical aspects and cytokine response in severe H1N1 influenza A virus infection. Crit Care 2010; 14:R203.

8 To KK, Hung IF, Li IW, et al. Delayed clearance of viral load and marked cytokine activation in severe cases of pandemic H1N1 2009 influenza virus infection. Clin Infect Dis 2010; 50:850-859.

9 Maines TR, Jayaraman A, Belser JA, et al. Transmission and pathogenesis of swine-origin 2009 A(H1N1) influenza viruses in ferrets and mice. Science 2009; 325:484-487.

10 Munster VJ, de Wit E, van den Brand JM, et al. Pathogenesis and transmission of swine-origin 2009 A(H1N1) influenza virus in ferrets. Science 2009; 325:481-483.

11 Sun Y, Bi Y, Pu J, et al. Guinea pig model for evaluating the potential public health risk of swine and avian influenza viruses. PLoS One 2010; 5:e15537.

12 Meunier I, Pillet S, Simonsen JN, von Messling V. Influenza pathogenesis: lessons learned from animal studies with $\mathrm{H} 5 \mathrm{~N} 1$, H1N1 Spanish, and pandemic H1N1 2009 influenza. Crit Care Med 2010; 38(4 Suppl):e21-e29.

13 Chan $\mathrm{KH}$, Zhang AJ, To KK, et al. Wild type and mutant 2009 pandemic influenza A (H1N1) viruses cause more severe disease and higher mortality in pregnant BALB/c mice. PLoS One 2010; 5:e13757.

14 Belser JA, Wadford DA, Pappas C, et al. Pathogenesis of pandemic influenza $\mathrm{A}(\mathrm{H} 1 \mathrm{~N} 1)$ and triple-reassortant swine influenza A (H1) viruses in mice. J Virol 2010; 84:4194-4203.

15 Mauad T, Hajjar LA, Callegari GD, et al. Lung pathology in fatal novel human influenza A (H1N1) infection. Am J Respir Crit Care Med 2010; 181:72-79.

16 Sun Y, Shi Y, Zhang W, et al. In silico characterization of the functional and structural modules of the hemagglutinin protein from the swine-origin influenza virus A (H1N1)-2009. Sci China Life Sci 2010; 53:633-642.

17 Gao GF, Sun Y. It is not just AIV: from avian to swine-origin influenza virus. Sci China Life Sci 2010; 53:151-153.

18 Quispe-Laime AM, Bracco JD, Barberio PA, et al. H1N1 influenza A virus-associated acute lung injury: response to combination oseltamivir and prolonged corticosteroid treatment. Intensive Care Med 2010; 36:33-41.

19 Hui DS, Lee N, Chan PK. Clinical management of pandemic 2009 influenza A(H1N1) infection. Chest 2010; 137:916-925.

20 Martin-Loeches I, Lisboa T, Rhodes A, et al. Use of early corticosteroid therapy on ICU admission in patients affected by severe pandemic $(\mathrm{H} 1 \mathrm{~N} 1) \mathrm{v}$ influenza A infection. Intensive Care Med 2011; 37:272-283.

21 Lee VJ, Yap J, Cook AR, et al. Oseltamivir ring prophylaxis for containment of $2009 \mathrm{H} 1 \mathrm{~N} 1$ influenza outbreaks. $N$ Engl $J$ Med 2010; 362:2166-2174.

22 Goldstein E, Cowling BJ, O'Hagan JJ, et al. Oseltamivir for treatment and prevention of pandemic influenza $\mathrm{A} / \mathrm{H} 1 \mathrm{~N} 1$ virus infection in households, Milwaukee, 2009. BMC Infect Dis 2010; 10:211.

$23 \mathrm{Yu} \mathrm{H}$, Liao Q, Yuan Y, et al. Effectiveness of oseltamivir on disease progression and viral RNA shedding in patients with mild pandemic 2009 influenza A H1N1: opportunistic retrospective study of medical charts in China. BMJ 2010; 341:c4779.

24 Steinman L. A brief history of $\mathrm{T}(\mathrm{H}) 17$, the first major revision in the $\mathrm{T}(\mathrm{H}) 1 / \mathrm{T}(\mathrm{H}) 2$ hypothesis of $\mathrm{T}$ cell-mediated tissue damage. Nat Med 2007; 13:139-145.

25 Cua DJ, Sherlock J, Chen Y, et al. Interleukin-23 rather than interleukin-12 is the critical cytokine for autoimmune inflammation of the brain. Nature 2003; 421:744-748.

26 Murphy CA, Langrish CL, Chen Y, et al. Divergent pro- and antiinflammatory roles for IL-23 and IL-12 in joint autoimmune inflammation. J Exp Med 2003; 198:1951-1957.

27 Park H, Li Z, Yang XO, et al. A distinct lineage of CD4 T cells regulates tissue inflammation by producing interleukin 17. Nat Immunol 2005; 6:1133-1141.

28 Hamada H, Garcia-Hernandez Mde L, Reome JB, et al. Tc17, a unique subset of CD8 T cells that can protect against lethal influenza challenge. J Immunol 2009; 182:3469-3481.

29 Crowe CR, Chen K, Pociask DA, et al. Critical role of IL17RA in immunopathology of influenza infection. J Immunol 2009; 183:5301-5310.

30 Kudva A, Scheller EV, Robinson KM, et al. Influenza A inhibits Th17-mediated host defense against bacterial pneumonia in mice. J Immunol 2011; 186:1666-1674.

31 Reichert JM, Rosensweig CJ, Faden LB, Dewitz MC. Monoclonal antibody successes in the clinic. Nat Biotechnol 2005; 23:1073-1078.

32 Zhu J, Paul WE. Heterogeneity and plasticity of T helper cells. Cell Res 2010; 20:4-12.

$33 \mathrm{Xu} \mathrm{S}$, Cao X. Interleukin-17 and its expanding biological functions. Cell Mol Immunol 2010; 7:164-174.

34 Chang SH, Dong C. Signaling of interleukin-17 family cytokines in immunity and inflammation. Cell Signal 2011; 23:1069-1075.

$35 \mathrm{Xu} \mathrm{G}$, Zhang L, Wang DY, et al. Opposing roles of IL-17A and IL-25 in the regulation of TSLP production in human nasal epithelial cells. Allergy 2010; 65:581-589.

36 Miossec P, Korn T, Kuchroo VK. Interleukin-17 and type 17 helper T cells. N Engl J Med 2009; 361:888-898.

37 Hueber W, Patel DD, Dryja T, et al. Effects of AIN457, a fully human antibody to interleukin-17A, on psoriasis, rheumatoid arthritis, and uveitis. Sci Transl Med 2010; 2:52ra72.

(Supplementary information is linked to the online version of the paper on the Cell Research website.) 